FERMILAB-PUB-93/098-A

April 1993

\title{
Hydrodynamic Detonation Instability in Electroweak and QCD Phase Transitions
}

\author{
Mark Abney* \\ Department of Astronomy and Astrophysics, The Enrico Fermi Institute, \\ The University of Chicago, Chicago, Illinois 60637 \\ and \\ NASA/Fermilab Astrophysics Center, \\ Fermi National Accelerator Laboratory, Batavia, Illinois 60510
}

\begin{abstract}
The hydrodynamic stability of deflagration and detonation bubbles for a first order electroweak and QCD phase transition has been discussed recently with the suggestion that detonations are stable. We examine here the case of a detonation more carefully. We find that in front of the bubble wall perturbations do not grow with time, but behind the wall modes exist which grow exponentially. We briefly discuss the possible meaning of this instability.
\end{abstract}

PACS number(s): 98.80.Cq, 95.30.Lz

*email: abney@rainbow.uchicago.edu 
A first order phase transition involves the nucleation of a bubble of one phase within a medium of the original phase. If the bubbles are large enough, they expand, collide and coalesce until the original phase has been completely replaced by the new phase. Though many of the details of this process are not definitively known - the expansion rate of the bubbles for instance - understanding the dynamics of such transitions may provide valuable insights into the conditions of the early universe.

Of particular interest are the electroweak (EW) and QCD phase transitions and the possible ramifications towards the generation of a baryon asymmetry in the EW case and the concentration of baryons in the QCD case. If the EW phase transition is first order, the possibility of baryon asymmetry generation arises [泪]. Furthermore, if the asymmetry is created by interaction with the bubble wall, it becomes important to understand in detail the shape and structure of the wall [2]. A first order QCD phase transition, on the other hand, may result in baryon concentrating effects [3]. Crucial to this is the concept of phase separation 四. The generation of a baryon concentration may depend on whether, and how effectively, the two phases mix during the period of bubble expansion. The stability of the bubble wall and the possible existence of turbulence are thus important factors when considering the effects of these phase transitions.

Recent investigations [ [ [, 5, 6] of the hydrodynamic stability of the bubble wall restrict their attention to the case of a deflagration front, namely a bubble wall which is propagating at a speed slower than sound relative to the old phase. Recent estimates support the assumption that the bubble wall will propagate subsonically [7]. Even if these estimates are correct, however, there may exist situations which allow for the supersonic propagation of the bubble, i.e., a detonation front. It was suggested by Kamionkowski and Freese [5] that the existence of an instability in the deflagration front could result in the acceleration of the front until it becomes a detonation, a phenomenon which is observed in laboratories studying combustion [8]. The paper by Huet et al. [6] analyzed the dispersion relation obtained for 
a perfect relativistic fluid and concluded that there could be no perturbations which grow in time in the detonation case. They, however, only examine the region in front of the expanding bubble and not the region behind it. In this paper we employ the standard linear stability analysis used by others [4, 6, 9, 10] to examine the possible existence of instabilities both in front of and behind the bubble wall in the detonation case.

Detonations and deflagrations consist of two different phases separated by a transition region referred to as the bubble wall. A detonation front propogates at a velocity greater than the local speed of sound relative to the original phase. Behind the wall, the medium has a velocity equal to or less than the speed of sound in the second phase (see Steinhardt's article [11] for a discussion of relativistic detonation and shock waves). We choose to move into the frame of the moving wall and position it at $x=0$. To the left of the wall $(x<0)$ is the original (e.g., quark) phase, while to the right $(x>0)$ is the new (e.g., hadron) phase. In region $1(x<0)$ the fluid has a positive velocity greater than that of sound $\left(v_{1}>c_{s 1}>0\right)$. In region $2(x>0)$ the fluid has a positive velocity less than that of sound $\left(0<v_{2}<c_{s 2}\right)[11$. The situation is illustrated in Figure 1. Because of the different velocities, the behavior of perturbations and their effect on the bubble wall will differ. Since perturbations cannot travel faster than the speed of sound, we expect that in region one they should be "swept away" as the fluid passes through the wall. This is in agreement with references [5, 6]. The situation behind the wall is considerably different, there being no a priori reason why one should expect perturbations to decay with time.

We first consider the behavior of a relativistic perfect fluid in the two separate regions. Using the metric $g=(+,-,-,-)$ the stress-energy of a perfect fluid is $T^{\mu \nu}=w u^{\mu} u^{\nu}-p g^{\mu \nu}$ where we have taken $c=1$, with $e$ the energy density, $p$ the pressure, and $w=e+p$ the enthalpy density. The equations of motion are:

$$
u^{\mu} \partial_{\mu} p+c_{s}^{2} w \partial_{\mu} u^{\mu}=0
$$




$$
w u^{\mu} \partial_{\mu} u_{\nu}-\partial_{\nu} p+u_{\nu} u^{\mu} \partial_{\mu} p=0
$$

where $u_{\mu}=(\gamma, \gamma \vec{v})$ and $\gamma=1 / \sqrt{1-v^{2}}$. We take perturbations in the velocity and pressure of the fluid about a constant solution:

$$
\begin{aligned}
& p=p_{0}+\delta p \\
& \vec{v}=\vec{v}_{0}+\delta \vec{v} \\
& \vec{v}_{0}=v_{0} \hat{x} \\
& \delta \vec{v}=\delta v_{x} \hat{x}+\delta v_{y} \hat{y} .
\end{aligned}
$$

Keeping terms up to first order in $\delta p$ and $\delta \vec{v}$, we can write the equations of motion as:

$$
\begin{aligned}
\left(1-c_{s}^{2} v_{0}^{2}\right) \frac{\partial}{\partial t} \delta p+v_{0}\left(1-c_{s}^{2}\right) \frac{\partial}{\partial x} \delta p+w c_{s}^{2}\left(\frac{\partial}{\partial x} \delta v_{x}+\frac{\partial}{\partial y} \delta v_{y}\right) & =0 \\
\frac{v_{0}}{w \gamma^{2}} \frac{\partial}{\partial t} \delta p+\frac{\partial}{\partial t} \delta v_{x}+\frac{1}{w \gamma^{2}} \frac{\partial}{\partial x} \delta p+v_{0} \frac{\partial}{\partial x} \delta v_{x} & =0 \\
\frac{\partial}{\partial t} \delta v_{y}+v_{0} \frac{\partial}{\partial x} \delta v_{y}+\frac{1}{w \gamma^{2}} \frac{\partial}{\partial y} \delta p & =0
\end{aligned}
$$

where (1) comes from the conservation of energy and (2)-(3) are the relativistic Euler's equations. It is more convenient to write this system as the matrix equation:

$$
\mathbf{A}_{\mathbf{t}} \frac{\partial}{\partial t} \vec{W}+\mathbf{A}_{\mathbf{x}} \frac{\partial}{\partial x} \vec{W}+\mathbf{A}_{\mathbf{y}} \frac{\partial}{\partial y} \vec{W}=0
$$

where

$$
\begin{gathered}
\vec{W}=\left(\begin{array}{c}
\delta p \\
\delta v_{x} \\
\delta v_{y}
\end{array}\right) \\
\mathbf{A}_{\mathbf{t}}=\left(\begin{array}{ccc}
1-c_{s}^{2} v_{0}^{2} & 0 & 0 \\
\frac{v_{0}}{w \gamma^{2}} & 1 & 0 \\
0 & 0 & 1
\end{array}\right) \mathbf{A}_{\mathbf{x}}=\left(\begin{array}{ccc}
v_{0}\left(1-c_{s}^{2}\right) & w c_{s}^{2} & 0 \\
\frac{1}{w \gamma^{2}} & v_{0} & 0 \\
0 & 0 & v_{0}
\end{array}\right) \mathbf{A}_{\mathbf{y}}=\left(\begin{array}{ccc}
0 & 0 & w c_{s}^{2} \\
0 & 0 & 0 \\
\frac{1}{w \gamma^{2}} & 0 & 0
\end{array}\right) .
\end{gathered}
$$

This system may be solved to obtain a solution of the form:

$$
\vec{W}(x, y, t)=\vec{F}(x) e^{-i(\omega t+k y)}
$$

where

$$
\vec{F}(x)=\sum_{j} a_{j} e^{-i q_{j} x} \vec{R}_{j} .
$$


The $a_{j}$ are constants, and $\vec{R}_{j}$ are eigenvectors. The $q_{j}$ are found by solving the characteristic equation for (4). Doing this, we obtain the dispersion relation

$$
\left(q v_{0}+\omega\right)\left[\frac{1}{c_{s}^{2}}\left(q v_{0}+\omega\right)^{2}-\left(q+v_{0} \omega\right)^{2}-\left(1-v_{0}^{2}\right) k^{2}\right]=0
$$

The solutions for $\mathrm{q}$ are:

$$
\begin{aligned}
& q_{1}=\frac{-\omega}{v_{0}} \\
& q_{2,3}=\frac{1}{v_{0}^{2}-c_{s}^{2}}\left[\left(c_{s}^{2}-1\right) v_{0} \omega \pm c_{s}\left(1-v_{0}^{2}\right) \sqrt{\omega^{2}+\frac{v_{0}^{2}-c_{s}^{2}}{1-v_{0}^{2}} k^{2}}\right] .
\end{aligned}
$$

These, (国)-(7), are the same as the equations obtained in reference [6] equations (32), (34)(36). It will be of future interest to note that in the case where $v_{0}=c_{s}$ we obtain two solutions, $q_{1}$ from above and

$$
q_{2}=\frac{-\left(1+c_{s}^{2}\right)}{2 c_{s}} \omega+\frac{c}{2} \frac{k^{2}}{\omega}
$$

Thus, the solution for perturbations in a perfect relativistic fluid is:

$$
\vec{W}(x, y, t)=\left(a_{1} \vec{R}_{1} e^{-i q_{1} x}+a_{2} \vec{R}_{2} e^{-i q_{2} x}+a_{3} \vec{R}_{3} e^{-i q_{3} x}\right) e^{-i(\omega t+k y)}
$$

where

$$
\vec{R}_{1}=\left(\begin{array}{c}
0 \\
1 \\
\frac{q_{1}}{k}
\end{array}\right) \text { and } \vec{R}_{j}=\left(\begin{array}{c}
1 \\
\frac{-\left(q_{j}+v_{0} \omega\right)}{w \gamma^{2}\left(\omega+v_{0} q_{j}\right)} \\
\frac{-k}{w \gamma^{2}\left(\omega+v_{0} q_{j}\right)}
\end{array}\right) \text { for } j=2,3
$$

We now wish to examine whether there exist any unstable modes of $\vec{W}$ (i.e., modes where Im $\omega>0$ ) which obey the boundary conditions $\vec{W} \rightarrow 0$ as $x \rightarrow \pm \infty$. First, consider region one $(x<0)$. In order to satisfy the boundary condition as $x \rightarrow-\infty$, we must require either $\operatorname{Im} q_{j}>0$ or $a_{j}=0$. However, with some algebra, we can show from (17) that if $\operatorname{Im} \omega>0$ then $\operatorname{Im} q_{j}<0$ and therefore $a_{j}=0$ for $j=1,2,3$. That is, perturbations which grow in time cannot exist in front of the bubble wall. This is the conclusion reached in reference [6]. Let 
us next consider what occurs in region two $(x>0)$. Here, the $x \rightarrow+\infty$ boundary condition requires either $\operatorname{Im} q_{j}<0$ or $a_{j}=0$. We find, if $\operatorname{Im} \omega>0$ then $\operatorname{Im} q_{1,2}<0$ and $\operatorname{Im} q_{3}>0$. Thus, behind the wall we need require only that $a_{3}=0$. That is, since we can satisfy the $x \rightarrow+\infty$ boundary condition without requiring $a_{1,2}=0$, the solution for the perturbations, equation (9), is not identically zero, i.e., perturbations behind the bubble wall that grow in time may exist.

The above work, however, is not enough to prove that instabilities do, in fact, occur. It is still necessary to impose the constraints of the boundary conditions across the bubble wall to determine whether the instabilities do not contradict the relevant conservation laws. Let us use notation where subscripts of one or two indicate quantities in region one $(x<0)$ or two $(x>0)$ respectively. Also, we assume that there exists a perturbation of the bubble shape of the form:

$$
\Delta(y, t)=D e^{-i(\omega t+k y)} .
$$

We require

- conservation of energy:

$$
w_{1} \gamma_{1}^{2} v_{1}=w_{2} \gamma_{2}^{2} v_{2}
$$

- conservation of momentum:

$$
w_{1} \gamma_{1}^{2} v_{1}^{2}+p_{1}=w_{2} \gamma_{2}^{2} v_{2}^{2}+p_{2}-\sigma\left(\frac{\partial^{2}}{\partial y^{2}}-\frac{\partial^{2}}{\partial t^{2}}\right) \Delta
$$

- continuity of transverse velocity:

$$
v_{1 y}+v_{1} \frac{\partial \Delta}{\partial y}=v_{2 y}+v_{2} \frac{\partial \Delta}{\partial y} .
$$

Recalling that $W=0$ in region one, the above equations may then be linearized to obtain in region two:

$$
\delta p=\frac{1}{\Gamma_{-}}\left[-2 \gamma_{2}^{2} w_{2} v_{2} \frac{v_{1}-v_{2}}{v_{1}}(-i \omega)+\sigma\left(\omega^{2}-k^{2}\right)\right] \Delta
$$




$$
\begin{aligned}
\delta v_{x} & =\frac{\Gamma_{+}}{\Gamma_{-}}\left(\frac{v_{1}-v_{2}}{v_{1}}\right)(-i \omega) \Delta+\left(1-\frac{\Gamma_{+}}{\Gamma_{-}}\right) \frac{\sigma\left(\omega^{2}-k^{2}\right)}{2 \gamma_{2}^{2} w_{2} v_{2}} \Delta \\
\delta v_{y} & =\left(v_{1}-v_{2}\right)(-i k) \Delta
\end{aligned}
$$

where $\Gamma_{ \pm}=1 \pm \gamma_{2}^{2} \theta_{2} v_{2}^{2}$ and $\theta_{2}=1+1 / c_{s 2}$. Then (10)-(12) give us the perturbations just interior to the bubble, i.e., as $x \rightarrow 0^{+}$. Since we require $a_{3}$ to be zero for $x>0$ we are left with $a_{1}, a_{2}$, and $D$ as undetermined constants. By matching the general solution of perturbations for $x>0$, equation (91), with equations (10)-(12), we obtain three equations for these three unknowns. We will always have a solution for such a system provided that the determinant of the coefficient matrix vanishes. Let us write equations (10)-(12) as

$$
\vec{Y} \Delta \equiv\left(\begin{array}{c}
\delta p \\
\delta v_{x} \\
\delta v_{y}
\end{array}\right) .
$$

Matching solutions (9) with (13) we get:

$$
\vec{W}\left(0^{+}, y, t\right)=\left(a_{1} \vec{R}_{1}+a_{2} \vec{R}_{2}\right) e^{-i(\omega t+k y)}=\vec{Y} D e^{-i(\omega t+k y)}
$$

This equation may be rearranged into a single $3 \times 3$ matrix equation where the columns of the matrix are the vectors $\vec{R}_{1}, \vec{R}_{2}$ and $\vec{Y}$.

$$
\left(\vec{R}_{1}\left|\vec{R}_{2}\right| \vec{Y}\right)\left(\begin{array}{c}
a_{1} \\
a_{2} \\
D
\end{array}\right)=0
$$

By taking the determinant of the above $3 \times 3$ matrix we obtain an equation in $\omega, q_{2}, k, \sigma / w_{2}$, $v_{1}, v_{2}$, and $c_{s 2}$ :

$$
\begin{aligned}
& \frac{1}{\Gamma_{-} v_{2} k\left(\omega+v_{2} q_{2}\right)} \times \\
& \quad\left(i \frac{v_{1}-v_{2}}{v_{1}}\left[\left(\Gamma_{+}-2 v_{2}^{2}\right) \omega^{3}+v_{2}\left(\Gamma_{+}-2\right) q_{2} \omega^{2}+\left(2 v_{2}-\Gamma_{-} v_{1}\right) v_{2} k^{2} \omega-\Gamma_{-} v_{1} v_{2}^{2} q_{2} k^{2}\right]\right. \\
& \left.\quad+\frac{\sigma\left(\omega^{2}-k^{2}\right)}{2 \gamma_{2}^{2} w_{2} v_{2}}\left[\left(\Gamma_{+}-\Gamma_{-}\left(1+2 v_{2}^{2}\right)\right) \omega^{2}+\left(\Gamma_{+}-3 \Gamma_{-}\right) v_{2} q_{2} \omega+2 \Gamma_{-} v_{2}^{2} k^{2}\right]\right)=0
\end{aligned}
$$

Combining equations (7) and (14) and solving for $\omega$ we can eliminate $q_{2}$ and obtain an equation for $\omega$ as a function of $v_{1}, v_{2}, c_{s 2}, \sigma / w_{2}$ and $k$. By studying this equation we 
can determine if there exist any cases where $\omega$ has a positive imaginary solution, thereby establishing that the detonation has a self-sustaining instability.

Of particular relevance is the case of a Chapman-Jouget detonation. This occurs when the velocity behind the wall is equal to the speed of sound $\left(v_{2}=c_{s 2}\right)$. Steinhardt showed that any detonation with spherical symmetry must be of the Chapman-Jouget type [11]. It has also been postulated that any "naturally" occuring detonation will meet this condition [10]. In order to examine this case we use the value for $q_{2}$ given in equation (8). It is interesting to look at the case where the surface tension is zero $(\sigma=0)$; there are four solutions to $\omega$, two of which are positive imaginary. The solutions are:

$$
\begin{aligned}
& \omega= \pm i \sqrt{\frac{c_{s 2} v_{1}}{2-c_{s 2}^{2}}} c_{s 2} k \\
& \omega= \pm i \sqrt{\frac{1}{1-c_{s 2}}} c_{s 2} k
\end{aligned}
$$

The full equation with surface tension cannot be solved analytically. Instead we look at the behavior of $\omega$ under different limits. In the long wavelength limit $(k \rightarrow 0)$, keeping terms up to second order in $k$, equation (14) becomes:

$$
\begin{aligned}
& \frac{3 \sigma\left(1-c_{s}^{2}\right)}{2 w_{2} c_{s}}\left(1-c_{s}^{4}\right) \omega^{5}+i \frac{v_{1}-c_{s}}{v_{1}}\left(c_{s}^{4}-3 c_{s}^{2}+2\right) \omega^{4}+\frac{\sigma\left(1-c_{s}^{2}\right)}{w_{2} c_{s}}\left(-c_{s}^{4}+\frac{3}{2}\left(c_{s}^{2}-1\right)\right) k^{2} \omega^{3} \\
& \quad+i\left(-c_{s}^{3} v_{1}-c_{s}^{2}+c_{s} v_{1}+2\right) c_{s}^{2} \frac{v_{1}-c_{s}}{v_{1}} k^{2} \omega^{2}=0
\end{aligned}
$$

Notice that the quantity $\sigma / w_{2}$ provides a natural length scale. In Figure 2, the behavior of $\operatorname{Im} \omega$ was plotted as a function of $k$ with values of $v_{1}=1.5 c_{s}$ and $c_{s}=1 / \sqrt{3}$. The particular choice for $v_{1}$ is arbitrary; the behaviour of $\operatorname{Im} \omega$ is not altered significantly for different values. We have chosen a solution which matches with an unstable mode in the zero surface tension case, and we see that the rate of expansion increases slightly slower than linearly with the wave number, an effect of the surface tension. The discontinuity which appears as $k$ increases is an artifact of the small $k$ approximation; its location approaches the origin as 
the surface tension is increased. That is, with a larger surface tension we must go to a larger wavelength in order to insure that the $k \rightarrow 0$ approximation is valid, as we expect.

Next, consider the short wavelength limit. As $k$ goes to infinity only higher orders of $k$ contribute and we get:

$$
\begin{aligned}
\frac{\sigma\left(1-c_{s}^{2}\right)}{w_{2} c_{s}}\left(-c_{s}^{4}+\frac{3}{2}\left(c_{s}^{2}-1\right)\right) k^{2} \omega^{3} & +i\left(-c_{s}^{3} v_{1}-c_{s}^{2}+c_{s} v_{1}+2\right) c_{s}^{2} \frac{v_{1}-c_{s}}{v_{1}} k^{2} \omega^{2} \\
& -\frac{\sigma\left(1-c_{s}^{2}\right)^{2}}{2 w_{2}} c_{s} k^{4} \omega+i\left(v_{1}-c_{s}\right) c_{s}^{5} k^{4}=0 .
\end{aligned}
$$

Figure 3 shows Im $\omega$ as a function of $k$ with the same values as in Figure 2. Here we notice that as $k$ gets larger Im $\omega$ quicky approaches a constant value of approximately $0.144 w_{2} / \sigma$. Unlike in the deflagration case, where the surface tension results in there being a lower limt cutoff in the wavelength of instabilites, with a Chapman-Jouget detonation the growth rate does not drop to zero with shorter wavelengths, but rather reaches a maximum positive value.

In order for these instabilities to be dynamically relevant the mode with the fastest growth time scale must be less than the time scale associated with the phase transition. The QCD transition lasts a time $t_{H} \sim 10^{-5}$ s and has a value of $\sigma / w_{2} \sim 1 \mathrm{fm}$ [4]. We showed above that Im $\omega$ reached a maximum value of $\sim 0.14 w_{2} / \sigma$, corresponding to a time of $\sim 2.3 \times 10^{-23} \mathrm{~s}$. Thus, there is ample time for the instabilities to mature.

The scales associated with the EW phase transition are much smaller. In this case, using the formulas and parameter values as given in reference [12], we have $\sigma \sim 0.09 T_{c}^{3}$ and $w_{2} \sim 40 T_{c}^{4}$ where $T_{c}$ is the critical temperature. With a critical temperature of $150 \mathrm{GeV}$ we get $\sigma / w_{2} \sim 3 \times 10^{-6} \mathrm{fm}$. This leads to a maximum value of $\operatorname{Im} \omega$ of $\sim 5 \times 10^{4} \mathrm{fm}^{-1}$ corresponding to a time of $\sim 6.7 \times 10^{-29} \mathrm{~s}$. Since the phase transition lasts a time $\sim 0.005 t_{H}$ [12], where the Hubble time is $t_{H} \sim 10^{-11} \mathrm{~s}$, we see that there is sufficient time for the instabilities to grow.

Our general picture of the instabilities, then, is the following. For very large wavelengths 
the instabilities grow only very slowly with time, vanishing as $\lambda$ approaches infinity. As the wavelength decreases $\operatorname{Im} \omega$ rapidly increases with smaller $\lambda$ until a maximum rate is reached. Thus, unstable modes exist at all wavelengths. Even though these calculations were done for a Chapman-Jouget type of detonation, it is unlikely that the existence and time scale of the instabilities is so sensitive to the velocity behind the wall that this picture would be very much altered should $v_{2}<c_{s}$. Additional work beyond the scope of this report would need to be done, however, to verify this assertion.

Note that these instabilities do not exist in front of the bubble wall, but rather behind it and at its surface. What, then, are the ramifications on the bubble wall and the fluid inside it? To answer this, let us examine similar types of instabilites which exist in the laboratory. D'yakov and others [9] have calculated the stability condition for shock waves in a classical non-relativistic fluid. Though a shock wave and detonation are not identical, the fluid dynamics are quite similar. It is possible to establish conditions in the laboratory which violate this stability requirement; that is, under certain circumstances there are unstable modes which exist on the surface of and behind the shock wave. Thompson et al. [13] have recently done experiments which violate this shock stability condition. In their report they show a series of photographs where we see shock waves transform from planar to a billowy cloud-like surface as the stability condition is violated. The surface of the wave is travelling faster than the speed of sound, and the instabilities do not propogate forward from the shock. The transformation of the shock surface from planar to irregular is described by them as a "transition to turbulence" of the fluid behind the shock.

It is possible that a similar situation exists with the instabilites described above. The growth of perturbations behind the bubble wall may result in turbulence and a highly irregular surface. Such effects may entail an alteration in the picture of the phase transition proceeding through the growth of uniform spherical bubbles. This may very well be relevant when considering the possibility of baryon generation and concentration in the EW and QCD 
phase transitions respectively.

Whether detonations actually do arise, though, is not yet known. A more highly first order transition would likely result in an increased possibility of detonations. Another mechanism could be the existence of instabilities in deflagrations as desribed in reference [5]. The above analysis, however, is limited to the linear regime and does not take into account any non-linear effects that may arise. The result of such effects may be to stablize the perturbations, as has been observed with deflagrations in the laboratory [8]. It remains to be seen whether non-linearities are indeed important.

I would like to thank E. Kolb and G. Starkman for helpful discussions and comments. This work was supported in part by the University of Chicago Physics Department, the DOE and NASA grant NAGW-2381 at Fermilab.

\section{References}

[1] V. A. Kuzmin, V. A. Rubakov, M. E. Shaposnikov, Phys. Lett. B 155, 36 (1985).

[2] M. Dine, P. Huet, R. Singleton, L. Susskind, Phys. Lett. B 257, 351 (1991); M. Dine, P. Huet, R. Singleton, Nucl.Phys. B375, 625 (1992).

[3] E. Witten, Phys. Rev. D 30, 272 (1984).

[4] B. Link, Phys. Rev. Lett. 68, 2425 (1992).

[5] M. Kamionkowski, K. Freese, Phys. Rev. Lett. 69, 2743 (1992).

[6] P. Huet, K. Kajantie, R. G. Leigh, B. H. Liu, L. McLerran, SLAC-PUB-5943 (1992).

[7] M. Dine, R. G. Leigh, P. Huet, A. Linde, D. Linde, Phys. Rev. D 46, 550 (1992); B. H. Liu, L. McLerran, N. Turok, Phys. Rev. D 46, 2668 (1992); S. Y. Khlebnikov, Phys. Rev. D 46, 3223 (1992). 
[8] Ya B. Zeldovich, G. I. Barenblatt, V. B. Librovich, G. M. Makhviladze, The Mathematical Theory of Combustion and Explosions (Plenum, New York, 1985).

[9] S. P. D'yakov, Zhur. Eksptl. i Theor. Fiz. 27, 288 (1954) (translation: Atomic Energy Research Establishment AERE Lib./Trans. 648, 1956); V. M. Kontorovich, Sov. Phys. Tech.Phys. 6, 1179 (1957); J. J. Erpenbeck, Phys. of Fluids 5, 1181 (1962).

[10] L. Landau, D. Lifshitz, Fluid Mechanics (Pergamon, New York, 1959).

[11] P. J. Steinhardt, Phys. Rev. D 25, 2074 (1982).

[12] K. Enqvist, J. Ignatius, K. Kajantie, K. Rummukainen, Phys. Rev. D 45, 3415 (1992).

[13] P. A. Thompson, G. C. Carofano, Y. G. Kim, J. Fluid Mech. 166, 57 (1986). 


\section{Figure captions}

Figure 1: Schematic of the bubble wall in the frame of the wall. The wall is located at $x=0$ with the old phase (e.g., quarks) in the half-plane $x<0$ and the new phase (e.g., hadrons) in the half plane $x>0$.

Figure 2: Perturbation growth rate $(\operatorname{Im} \omega)$ as a function of wave number $(k)$ in the limit $k \rightarrow 0$, plotted with parameter values $c_{s}=1 / \sqrt{3}, v_{1}=1.5 c_{s}$ in units of $w_{2} / \sigma$.

Figure 3: The same as in Figure 2, but in the limit $k \rightarrow \infty$. 
This figure "fig1-1.png" is available in "png" format from: http://arxiv.org/ps/astro-ph/9305021v1 
This figure "fig1-2.png" is available in "png" format from: http://arxiv.org/ps/astro-ph/9305021v1 
This figure "fig1-3.png" is available in "png" format from: http://arxiv.org/ps/astro-ph/9305021v1 